\title{
Relation of Beat-to-Beat Variability to Heart Rate in Normal Sleeping Infants
}

\author{
N. M. MAZZA, M. A. F. EPSTEIN, G. G. HADDAD, H. S. LAW, R. B. MELLINS, AND \\ R. A. EPSTEIN ${ }^{(29)}$ \\ Departments of Anesthesiology, Chemical Engineering and Applied Chemistry, and Pediatrics, Columbia University, \\ New York, New York, USA
}

\begin{abstract}
Summary
The relationship of beat-to-beat heart rate variability (delta RR) and instantaneous heart rate was studied in eight normal infants while asleep during the first four months of life. The sleep state (REM or quiet) was determined using neurophysiologic and behavioral criteria. The results of regression analyses indicated that the delta $R R$ values were positively correlated with the instantaneous heart rate ( $R R$ intervals). The correlation coefficient range was 0.49 to 0.92 in quiet sleep and 0.50 to 0.93 in REM sleep. Regression analyses supported a linear approximation of the delta $R R$ to $R R$ relation over the $R R$ range investigated (400 to 520 msec). The median slope was 0.124 in REM and 0.117 in quiet sleep. The slopes of these linear functions were similar in both sleep states and at all ages. If beat-to-beat variability is to be used as an index of the integrity of the autonomic nervous system, these results suggest that delta $R R$ be corrected for $R R$. $A$ model is presented which relates the demonstrated positive correlation of delta RR to RR with the physiology of cardiac output control.
\end{abstract}

\section{Speculation}

The positive correlation of delta $R R$ with $R R$ is consistent with the view that beat-to-beat heart rate variability is a homeostatic mechanism required for control of cardiac output.

Beat-to-beat or short-term heart rate variability (delta $R R$ ) is used for identification of fetal distress and is believed to reflect the integrity of the autonomic nervous system in the fetus and newborn $(6,11,18)$. Autonomic dysfunction may be manifested by decreased short-term variability as seen in hypoxic newborn infants at delivery (15) and in infants of mothers given parasympatholytic drugs $(7,8,13,14)$.

Short-term variability may also prove to be a useful parameter for monitoring autonomic function during early infancy. However, as in the fetus $(7,8)$, short-term variability of the heart rate might be a function of the instantaneous heart rate (instantaneous heart rate defined as the inverse of the $R R$ interval of the electrocardiogram, i.e., $1 / R R$ ) during the first several postnatal months. If this were the case, the delta RR should be normalized to the instantaneous heart rate. Therefore, we investigated the relation between delta $R R$ and $R R$. Because heart rate and delta $R R$ change with age and sleep state (12), the effects of age and sleep state on the delta $R R$ to $R R$ relation were also studied.

\section{MATERIALS AND METHODS}

Studies were performed on eight normal infants during the first four months of life while asleep as part of a larger study on maturational changes in cardiorespiratory control mechanisms of early life. All the infants were the products of normal pregnancies (gestational age range, 38 to $42 \mathrm{wk}$; birth weight range, 2.7 to 4.5 $\mathrm{kg}$ ) and had normal growth and development. Twelve lead elec- trocardiograms done after each study were normal. The procedures and objectives of the study were explained to the parents, and informed consent was obtained. A total of four or five studies, lasting one to two hr, were performed on each infant while it was asleep. Serial studies were performed during the first two wk of life and subsequently at monthly intervals through four months of age. Each study was done in a quiet laboratory environment after a usual midmorning feeding.

\section{MEASUREMENT OF THE RR INTERVALS}

Electrocardiogram surface electrodes were positioned on the chest to obtain a unidirectional polygraph recording with a single prominent $\mathbf{R}$ wave which had a sharp peak. Each $\mathbf{R} \mathbf{R}$ interval of this wide-band electrocardiogram signal $(0.5$ to $2000 \mathrm{~Hz})$ was measured with an accuracy of $200 \mu \mathrm{sec}$ using a digital preprocessor designed in this laboratory (17). The data was carefully reviewed, and less than $0.1 \%$ of the 10,000 to 15,000 of each study RR intervals were rejected as artifact.

\section{SLEEP STAGING}

Surface electrodes were placed to record the neurophysiologic signals necessary to determine sleep state. These were: A C4-Al electroencephalogram, a single bipolar electrooculogram, and a submental electromyogram. All of these signals were recorded on the polygraph using low noise $(<2 \mu \mathrm{V}$ peak to peak) amplifiers. Sleep staging was performed visually for 30 -sec epochs by two independent observers using the electroencephalogram, electrooculogram, electromyogram, and behavioral criteria (1). All RR intervals during a given sleep state were included in the analysis.

\section{DATA ANALYSIS}

The absolute difference between the RR intervals of successive heart beats was used as a measure of short-term variability. It was calculated as

$$
\left|\Delta \mathbf{R}_{(\mathbf{n})}\right|=\left|\mathbf{R}_{(\mathbf{n}+1)}-\mathbf{R} \mathbf{R}_{(\mathbf{n})}\right|
$$

where $R R$ is the time in milliseconds from the peak of one $R$ wave to the next, and " $n$ " the interval number.

For each study, the $R R$ interval range was divided into tenmsec cells or bins, and the average value of the delta $R R$ for each RR bin was calculated. Only those RR bins which contained at least 10 delta $R R$ values were considered. This approach provided an estimate of the expected delta $R R$ value for each $R R$ bin and allowed us to study the nature of the relation. Regression analysis was performed on this average delta $R R$ as a function of $R R$ for each individual study for REM and quiet sleep.

The effects of age and sleep state on the delta RR to RR relationship were then investigated. For this purpose, the average delta RR's representing each bin in all studies at each age and sleep state were pooled, and regression analyses were done. This 
pooled data was analyzed for RR values between 400 and 520 msec (corresponding to heart rates of 150 and 115 , respectively). This range covered the average heart rate \pm 1 S.D. during the first four months of life (12). To characterize the slope of the regression relationship for any age and sleep state, we calculated the median of such slopes. The median was used rather than the mean to diminish the effects of outliers.

\section{RESULTS}

The results of regression analysis on the data for each sleep state in each study indicated that the delta RR values were positively correlated with the $\mathbf{R} R$ intervals. A typical plot of delta $\mathbf{R} R$ versus RR for one infant is shown in Figure 1. To determine whether the relation could be approximated by a linear function, we calculated the correlation coefficients of the delta $R R$ to $R R$ relationship. We considered the relation to be approximately linear if the correlation coefficient exceeded that threshold value $(r)$ necessary to satisfy $t=r \sqrt{n-2} / \sqrt{1-r^{2}}$ for $P<0.05$ (9). In 25 of 36 studies during quiet and 32 of 36 studies during REM sleep, the correlation coefficients were greater than these threshold values. The coefficients which were greater than the threshold values had a range of 0.49 to 0.92 in quiet sleep and a range of 0.50 to 0.93 in REM sleep.

To confirm the linearity of the relation of delta $R R$ to $R R$, polynomial regression analysis was performed on these studies in both REM and quiet sleep. The results of this analysis also supported a linear model because, when second-and third-order regressions were considered, the improvement in the sum of squares of the deviation from regression was less than $5 \%$ in all studies where the correlation coefficient exceeded the threshold value.

In all 25 studies during REM and 32 studies during quiet sleep where the relationship could be considered linear, the slopes of the least-square linear regression functions were positive. The median slopes in these studies were 0.124 (interquartile range, 0.090 to 0.170 ) for REM and 0.117 (interquartile range, 0.081 to 0.214 ) for quiet sleep. In one-half of the cases, the slopes were greater in REM, and in the other one-half, the slopes were greater in quiet sleep and hence could not be considered different (sign test, $P>0.25$ ). Therfore, there was not consistent trend with sleep state.
The data for each age were pooled to characterize the relation between delta RR and RR at each age. Figure 2 shows the linear regression lines of delta $R R$ versus $R R$ for the pooled data at each age in quiet and REM sleep, respectively. The slopes of these linear functions are similar, and these lines lie within the same narrow band (shaded areas) in both sleep states and at all ages.

To characterize the effect of sleep state on the delta RR-RR relation, the correlation coefficients of the pooled data for each age and sleep state were compared to their respective threshold values (calculated as defined previously). These correlation coefficients had a range of 0.42 to 0.61 in quiet sleep and 0.39 to 0.66 in REM sleep and are lower than those of individual states because they reflect intersubject differences. However, all exceeded their threshold values, further supporting approximation of the delta RR-RR relation by a linear function. Combining the pooled data for REM and quiet sleep at each age yielded correlation coeffi-

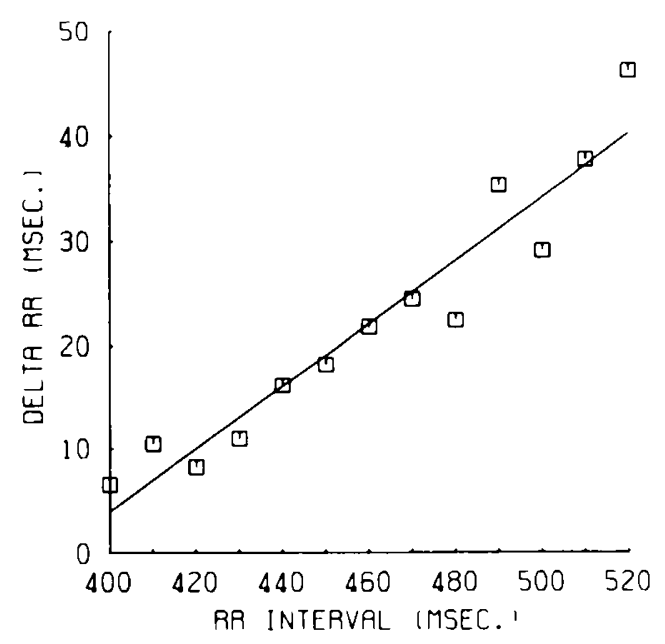

Fig. 1. Typical delta $R R$ versus $R R$ for one normal infant at age four months in REM sleep. The average delta $R R$ values for each $10 \mathrm{msec} R R$ cell or bin are plotted. The linear regression line through these points is shown.
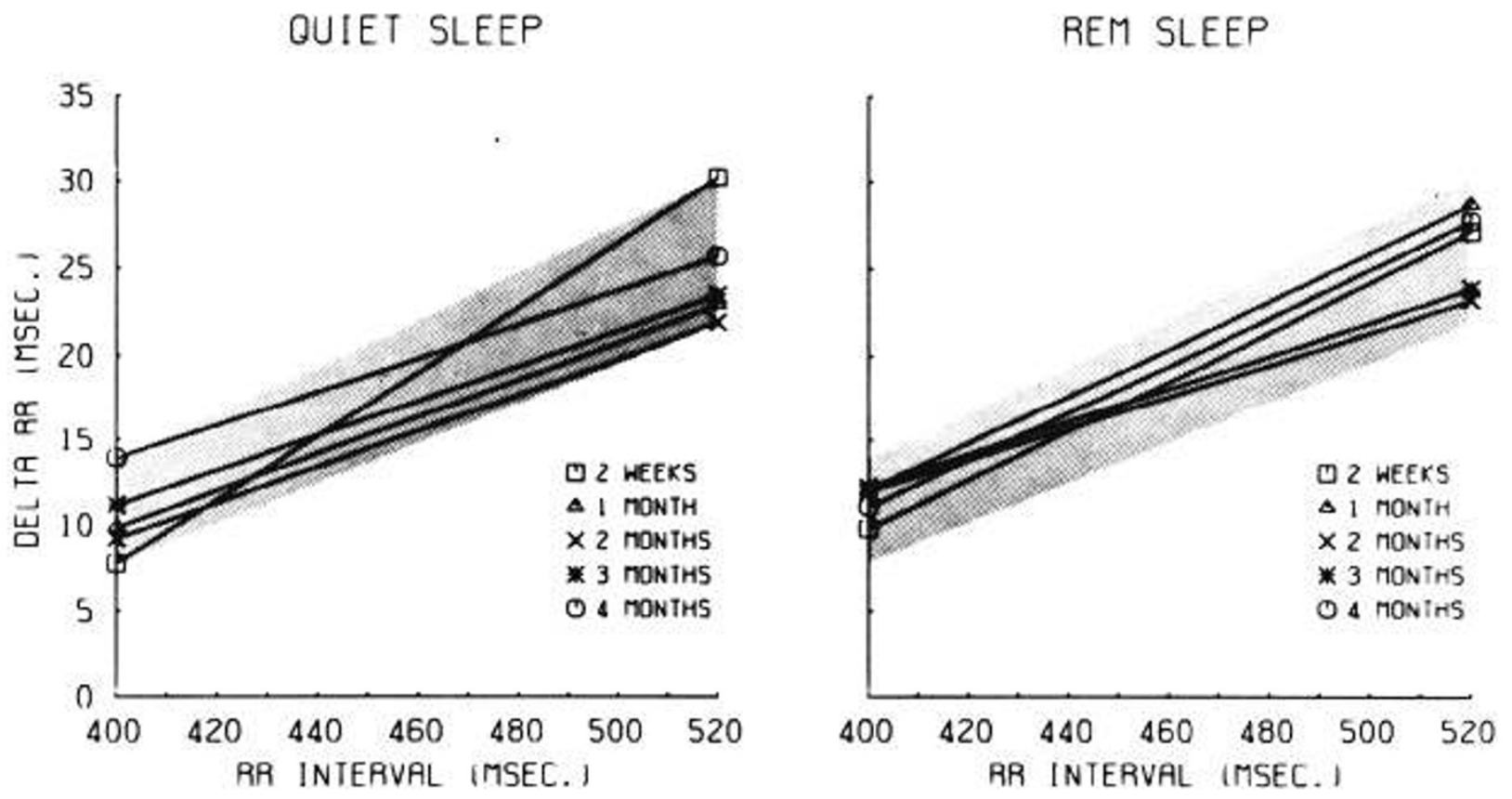

Fig. 2. The linear regression lines for delta RR versus RR at each age are shown for quiet (left) and REM (right) sleep. All of these regression lines for REM and quiet sleep fit into the same narrow range indicated by the shaded area in both plots. 
cients, slopes, and intercepts which were similar to those found for each sleep state alone. Indeed, the range of correlation coefficients for the combined data was 0.40 to 0.62 . Thus, to a first approximation, the delta $R R-R R$ relation may be considered independent of sleep state.

\section{DISCUSSION}

Our results show that short-term variability (delta $\mathbf{R} \mathbf{R}$ ) is positively related to instantaneous heart rate and may be approximated by a linear function for the RR range investigated ( 400 to 520 msec). This relation implies that delta $R R$ measurements should be corrected for heart rate because delta $R R$ depends not only on the activity and integrity of the autonomic nervous system (21, 23-26) but also on the heart rate itself. This is analogous to the interpretation of the QT interval (4). The delta $R R$ versus $R R$ relationship we have demonstrated does not appear to be influenced by sleep state or age. This implies that this striking relationship is resistant to maturational changes in heart rate and beat-tobeat variability (12), to postnatal developmental changes in the autonomic innervation of the heart (10), and to fluctuations in autonomic activity associated with various sleep states $(2,3,5)$. The linear relations we have used appear to adequately describe the delta $R R$ versus $R R$ relation for the heart rate range normally seen. The relation is, of course, not truly linear over a broader range because the regression lines intersect the $R R$ interval axis at approximately $300 \mathrm{msec}$, implying that there would be no variability beyond that point. If there were no variability, the heart rate would be fixed, which is clearly not the case under normal circumstances.

If beat-to-beat variability is to be used as an index of the integrity of the autonomic nervous system $(6,11,18)$, the results of this study suggest that correction of this index for heart rate may be desirable. This correction may be most important in those clinical situations where there is a wide range of heart rate.

The positive correlation of delta $R R$ to $R R$ may depend on the way cardiac output is regulated. Cardiac output (CO) under normal circumstances is adjusted primarily by heart rate (HR) rather than stroke volume (SV) changes $(16,19,20,22,27)$. Under conditions where SV is constant, the heart rate may be considered a measure of cardiac output since

$$
\mathrm{CO}=\mathrm{SV} \times \mathrm{HR}
$$

The relationship of instantaneous heart rate or cardiac output to the RR interval is hyperbolic (Fig. 3). From this model, one can predict that for a fixed increment in CO change, a larger delta $R R$ is necessary at a higher $R R$. By substituting $1 / R R$ for $H R$ in equation 1 above, and considering SV to be constant, one can show that

$$
\mathrm{dRR}=-\mathrm{RR}^{2} / \mathrm{SV} \times \mathrm{dCO}
$$

or

$$
|\Delta \mathrm{RR}|=\mathrm{RR}^{2} / \mathrm{SV} \times|\Delta \mathrm{CO}|
$$

If $\Delta C O$ is independent of $R R$, this model predicts that delta $R R$ should be positively correlated with RR. Figure 3 demonstrates this relationship. Our empirical finding tht delta $R R$ is positively correlated with $R R$ suggests that short-term heart rate variability may indeed represent attempts to control cardiac output in an incremental fashion.

\section{REFERENCES AND NOTES}

1. Anders, T., Emde, R., and Parmelee, A.: A Manual of Standardized Terminology, Techniques and Criteria for Scoring of States of Sleep and Wakefulness in Newborn Infants. ULCA Brain Information Service/BRI Publications Office. Los Angeles, CA (1971).

2. Ashton, R., and Connolly, K.: The relation of respiration rate and heart rate to sleep states in the human newborn. Dev. Med. Child. Neurol., 13: 180 (1971)

3. Baust, W., and Bohnert, B.: The regulation of heart rate during sleep. Exp. Brain Res., 7: 169 (1969).

4. Bazett, H. C.: An analysis of the time relations of electrocardiograms. Heart, 7: 353 (1920).

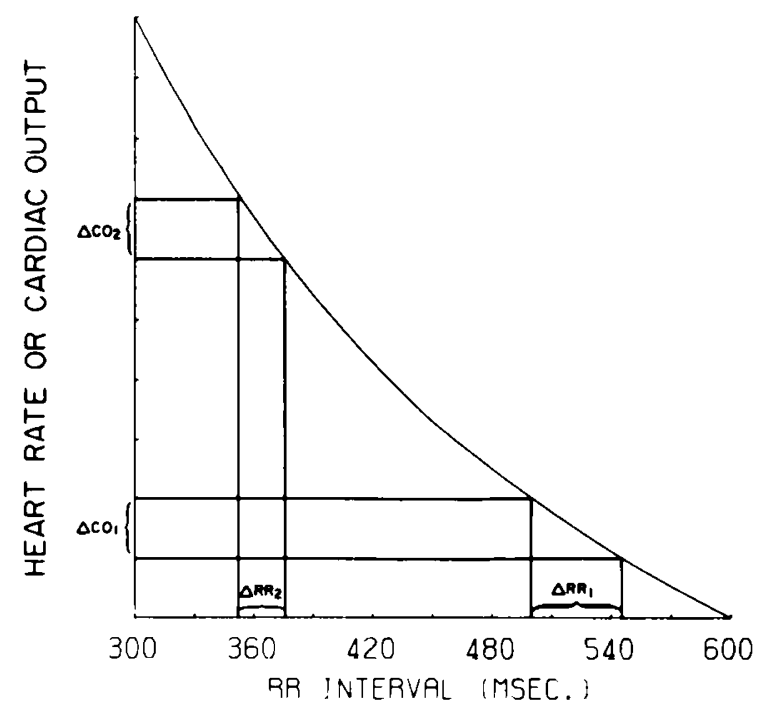

Fig. 3. Heart rate or cardiac output versus $R \mathbf{R}$ interval. Case 1 shows the relation of delta $C O$ and delta $R R$ at a low heart rate; case 2, at a high heart rate. For equal cardiac output changes $(\Delta C O)$, a larger delta $R R$ is required at a lower heart rate (higher $\mathbf{R} R$ ).

5. Bond, W. C., Bohs, C.. Ebey, J., Jr., and Wolf, S.: Rhythmic heart rate variability (sinus arrhythmia) related to stages of sleep. Cond. Reflex, 8: 98 (1973).

6. Church, S. C., Morgan, B. C., Oliver. T. K., Jr., and Guntheroth. W. G.: Cardiac arrhythmias in premature infants: an indication of autonomic immaturity? J. Pediatr., 71: 542 (1967).

7. DeHaan. J.: Fetal monitoring by physical methods. II. Interpretation of phenomena. Eur. J. Obstet. Gynecol. Reprod. Biol., 3: 95 (1973).

8. DeHaan, J., VanBemmel, J. H., Stolte, L. A. M., Veth, A. F. H., Janssens, J., and Eskes. T. K. A. B.: Trend detection in the fetal condition. Int. J. Gynaecol Obstet., 10: 202 (1972).

9. Edwards, A. L.: An Introduction to Linear Regression and Correlation. p. 84 (W. H. Freeman and Co., San Francisco, 1976).

10. Friedman, W. F., Pool, P. E., Jacobowitz, D., Seagren, S. C., and Braunwald, E.: Sympathetic innervation of the developing rabbit heart. Circ. Res., 23: 25 (1968).

11. Glick, G., and Brunwald, E.: Relative role of the sympathetic and parasympathetic nervous systems in the reflex control of heart rate. Circ. Res., 16: 363 (1965).

12. Harper, R. M., Hoppenbrouwers, T., Sterman, M. B., McGinty, D. J., and Hodgman, J.: Polygraphic studies of normal infants during the first six months of life. I. Heart rate and variability as a function of state. Pediatr. Res., 10: 945 (1976).

13. Hon, E. H.: Electronic evaluation of the fetal heart rate. VI. Fetal distress-a working hypothesis. Am. J. Obstet. Gynecol., 83: 333 (1962).

14. Hon, E. H., Bradfield, A. H., and Hess, O. W.: The electronic evaluation of the fetal heart rate. V. The vagal factor in fetal bradycardia. Am. J. Obstet. Gynecol., 82: 291 (1961).

15. Hon, E. H., and Lee, S. T.: Electronic evaluation of fetal heart rate. VIII. Patterns preceding fetal death. Am. J. Obstet. Gynecol., 87: 814 (1963).

16. Khatri, I. M., and Freis, E.: Hemodynamic changes during sleep. J. Appl. Physiol., 22: 867 (1967).

17. Law, H. S., Epstein, R. A., and Epstein, M. A.: High resolution determination of the RR interval. Am. J. Physiol., 236: H894 (1979).

18. Robinson, B. F., Epstein, S. E., Beiser, G. D., and Braunwald, E.: Control of heart rate by the autonomic nervous system. Circ. Res., 19: 400 (1966).

19. Rudolph. A. M.. and Heymann, M. A.: Fetal and neonatal circulation and respiration. Annu. Rev. Physiol., 36: 187 (1974).

20. Rudolph, A. M., and Heymann, M. A.: Cardiac output in the fetal lamb. The effects of spontaneous and induced changes of heart rate on right and left ventricular output. Am. J. Obstet. Gynecol., 124: 183 (1976).

21. Rudolph, A. J. Vallbona, C., and Desmond, M.: Cardiodynamic studies in the newborn. III. Heart rate patterns in infants with idiopathic respiratory distress syndrome. Pediatrics, 36: 551 (1965).

22. Rushmer, R. F.: Constancy of stroke volume in ventricular response to exertion. Am. J. Physiol., 196: 745 (1959).

23. Urbach, J. R., Phuvichit, B., Zweizig, H., Millican, E., Carrington, E. R., Loveland, M., Williams, J. M., Lambert, R. L., Duncan, A. M., Farrell, S. L., Simons, P. O., and Spurgeon, I. L.: Instanteous heart rate patterns in newborn infants. Am. J. Obstet. Gynecol., 93: 965 (1965).

24. Valimaki, I.: Postnatal alterations of cardiac rhythm. Clin. Pediatr., 9: 652 (1970).

25. Valimaki, I.: Heart rate variation in full term newborn infants. I. Biol. Neonat., 18: 129 (1971).

26. Warner, H. R., and Russell, R. O.: Effect of combined sympathetic and vagal stimulation on heart rate in the dog. Circ. Res., 24: 567 (1969).

27. Warner, H. R., and Toronto, A. F.: Regulation of cardiac output through stroke volume. Circ. Res., 8: 549 (1960). 
28. We acknowledge the critical review of the manuscript, especially the statistical analysis, by Professor Tze Lai. Department of Mathematical Statistics, Columbia University, New York.

29. Requests for reprints should be addressed to: Dr. Ralph A. Epstein. Associate Professor, Department of Anesthesiology, Presbyterian Hospital, 622 West 168
Street, New York, New York 10032 (USA)

30. This research was supported in part by NIH Contract HD 52856 and Training Grant HL 06012.

31. Received for publication January $22,1979$.

32. Accepted for publication April 4, 1979.

Copyright $\odot 1980$ International Pediatric Research Foundation. Inc $0031-3998 / 80 / 1403-0232 \$ 02.00 / 0$ 